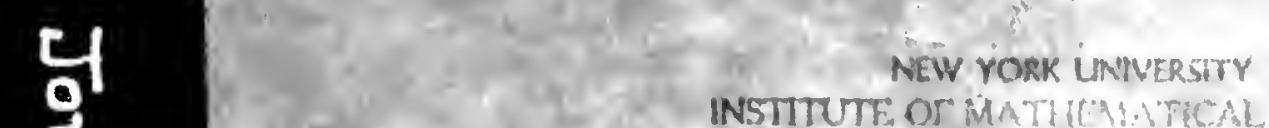

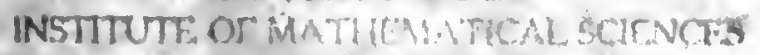

25 Wavent Pisce, New York, 3, M. Y. 



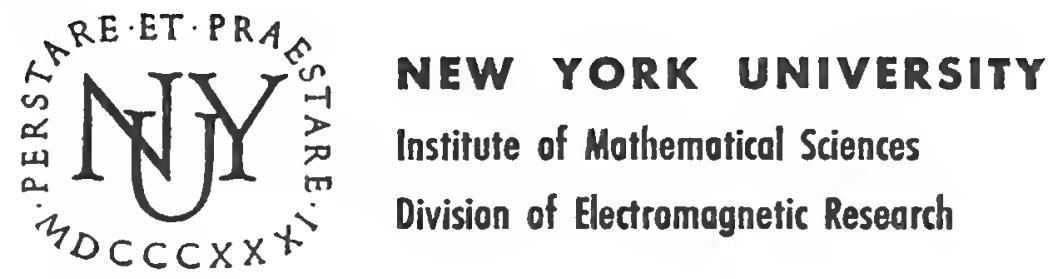

RESEARCH REPORT NO. EM-78

\section{A Critique of the Variational Method in Scattering Problems}

D. S. JONES

CONTRACT NO. AF- $19(122)-42$

MAY 1955 
NEW YORK UNIVERSITY

Institute of Mathematical Sciences

Division of Electromagnetic Research

Research Report No. EM-78

A CRITIQUE OF THE VARIATIONAL METHOD

IN SCATTERING PROBLEMS

D. S. Jones

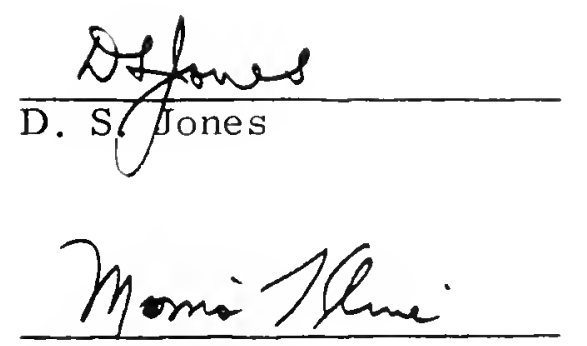

Morris Kline

Project Director

The research reported in this document has been made possible through support and sponsorship extended by the Air Force Cambridge Research Center, under Contract No. AF 19(122)-42. It is published for technical information only and does not necessarily represent recommendations or conclusions of the sponsoring agency. 

Abstract

It is shown that the variational method of dealing with the integral equations of scattering problems is equivalent to solving the integral equation directly by Galerkin's method and using the standard formule for the amplitude of the scattered wave. The second method also satisfies the reciprocity theorem. It is therefore suggested that the reciprocity theorem be used as the basis of approximation without the introduction of variational formulas.

The error involved in using an approximate solution is discussed and it is shown that only a special set of approximations can lead to accuracy at low frequencies. Some ways in which bounds for the error may be obtainod in special problems are also given.

\section{Table of Contents}

\section{Page}

1. Introduction

2. The equivalence of the variational method and Galerkin's method

3. The accuracy of the approximation

4. The low frequency behavior

5. Bounds for the error 



\section{Introduction}

The fact that an integral equation can be formulated as a variational principle was stated as early as 1884 by Volterra[1] but it has not been used for practical calculation in scattering problens until recent years when it was discovered by Schwinger that the amplitude of the scattered field is closely related to the quantity whose variation has to be considered. (In connection with small-amplitude sound waves, see Levine and Schwinger [2], and in connection with atomic collisions see Schwinger's results as quoted by Kohn [3]). The essence of the variational method is that, if a good approximation for the field is inserted in the variational expression, an improved approximation for the scat-tered amplitude should result. One particular way of choosing the approximation, that given by Levine and Schwinger [2], is to expand the field in a set of functions and solve the simultaneous linear equations resulting from the variational principle for the coefficients. It will be shown in the following that this is exactly equivalent to Galerkin's metinod of solving the integral equation and, furthermore, that only by expanding the field in a special set of functions can accuracy be obtained. Also this solution satisfies the reciprocity theorem.

Another way of approximation is to choose for the field an expression which is physically plausible and/or mathematically simple and insert it immedlately in the variational expression. It will be shown that this is equivalent to choosing an approximation which satisfies the reciprocity theorem.

It appears, therefore, that fundamentally the use of the variational method is to ensure that the reciprocity theorem is complied with. Since the reciprocity theorem is easily written down, it is obviously simpler to formulate our analysis in terms of it than to introduce the variational principle. The advantage is mainly one of clarity for, once the equation(s) to determine the approximation have been obtained, the Labor required to calculate the scattered amplitude is 
the same by either method.

2. The equivalence of the variational method and Galerkin's method ${ }^{\dagger}$

The mathematical problem encountered in scattering may be stated as follows: given a function $f$ and operator $L$, find a function $g$ such that

$$
f=\operatorname{Lg}
$$

The function $f$ is determined by the incident field and $g$ can be regarded as a suitable source distribution. The equation is supposed to have a unique solution. Examples of equation (1) are

$$
f\left(\underline{r}^{\prime}\right)=\int_{S} g(\underline{r}) \mathbf{I}\left(\underline{r}, \underline{r}^{\prime}\right) d \underline{r}
$$

for one of the boundary conditions in diffraction by an aperture, and

$$
f\left(\underline{r}^{\prime}\right)=g\left(\underline{r}^{\prime}\right)+\int_{S} V(\underline{r}) g(\underline{r}) \Psi\left(\underline{r}, \underline{r}^{\prime}\right) d \underline{r}
$$

for atomic collistons or scattering by a dielectric obstacle. In these equations

$$
I\left(\underline{r}, \underline{r}^{\prime}\right)=\frac{e^{-i k\left|\underline{r}-\underline{r^{\prime}}\right|}}{4 \pi\left|\underline{r}-\underline{r}{ }^{\prime}\right|} \text {, }
$$

$S$ is tre area of the aperture, $T$ is the volume of the scatterer and $T$ is the potential of the scattering particle. (For the dielectric obstacle $V$ is essentially a measure of the dielectric constant.) $)^{*}$

Often it is the scattered field at some distance from the obstacle and not $B$ itself which is of primary concern. The amplitude $A$ of the scattered field may be written

Sorte results similar to those of this section have been obtalned independently by Lippmann [4].

* Eq. (I) and the following analysis are applicable to vector field problems on regarding $f$ and $g$ as vectors and $L$ as a vector operator but no specific example will be quoted. 
$-3-$

(2)

$$
A=\left(f^{\prime}, g\right)=\left(g, f^{\prime}\right)
$$

where $f^{\prime}$ is some suitable incident field and $\left(f^{\prime}, g\right)$ means the inner product of $f^{\prime}$ and $g$. For example, the amplitude of the far field in the direction of the unit vector $\underline{n}$ is obtained correctly if we define $\left(f^{\prime}, g\right)$ by

$$
\left(\underline{f}^{\prime}, g\right)=\int_{S} f^{\prime}(\underline{r}) g(\underline{r}) d \underline{r}
$$

for the aperture problem or by

$$
\left(\underline{f}^{\prime}, g\right)=\int_{T} f^{\prime}(\underline{r}) V(\underline{r}) g(\underline{r}) d \underline{r}
$$

for the atomic collision and take $f^{\prime}=e^{i k \underline{x}} \cdot \underline{n}$. (A different choice of $f^{\prime}$ would lead to some other quantity, the near field for example, but would not invalidate the following analysis provided that it was a possible incident field.)

Let $g^{\prime}$ be such that

$$
f^{\prime}=I g^{\prime} \text {. }
$$

Assume that $I$ is a symmetric operator such that

$$
\left(h_{1}, I h_{2}\right)=\left(h_{2}, I h_{1}\right)
$$

Then, from (3), (4) and (1),

$$
\begin{aligned}
\left(f^{\prime}, g\right) & =\left(g^{\prime}, g\right) \\
& =\left(g^{\prime}, I g\right) \\
& =\left(g^{\prime}, f\right)
\end{aligned}
$$

or

$$
A=\left(f^{\prime}, g\right)=\left(f, g^{\prime}\right)=\left(g^{\prime}, \text { Lg }\right) \text {. }
$$

This is the reciprocity theorem which exists for most scattering problems. 
By an obvious use of this theorem, we can write

(6)

$$
A=\frac{\left(f^{\prime}, g\right)\left(f_{2} g^{\prime}\right)}{\left(g, I g^{\prime}\right)}
$$

It can be confirmed that the necessary and sufficient condition for (1) and (3) to be satisfied is that this expression for A is stationary for independent small variations of $\mathrm{g}$ and $\mathrm{g}^{\prime}$ about their correct values. Other variational principles are available (see, for example, Kohn [3]) but will not be considered here.

To demonstrate that the choice of an approximation to make (6) stationary is equivalent to Galerkin's solution of (I) we shall consider first the special case in which $f=f^{\prime}$ so that $g=g^{\prime}$. This corresponds, for example, to the problem of the circular aperture in a normally incident plane sound wave with the scattering observed in the direction of propagation of the incident wave. In this spectal case

$$
A=\frac{(f, g)^{2}}{(g, I g)} .
$$

Let $G_{0}, G_{1}, \ldots$ be a set of functions each of which is independent of the others and each of which satisfies any conditions, e.g., edge conditions, imposed on $g$. If an infinite number of $G_{0}, G_{1} \ldots$ is used, the set is also required to be complete. Now try an approximation $G$ to $g$ given by

$$
G=\sum_{n} c_{n} a_{n}
$$

where the sum may involve either a finite or infinite number of terms and the constants $c_{n}$ are to determined. We shall now prove the following 
Theorem 1 The necessary and sufficient conditions for the expressIon in (7) to be stationary for small variations of $G$ are

$$
\left(f, G_{n}\right)=\left(G_{n}, L G\right) \quad(n=0,1, \ldots) .
$$

$G$ then satisfies $(f, G)=(G, L G)$ and $A=(f, G)$.

The importance of this theorem lies in the fact that equations (9) are precisely those obtained by substituting the expansion (8) in (1) and taking the inner product of both sides with $G_{n}$, which is Galerkin's process. Then A may be calculated directly from its original definition (2) because the approximation satisfies the reciprocity theorem. Obviously this direct method of setting up the equations is to be preferred to determining them via the variation principle. It should be remarked that, if we make a one-term approximation so that $G=C_{0} G_{0}$, the reciprocity theorem is satisfied if $\left(f, G_{0}\right)=c_{0}\left(G_{0}, I G_{0}\right)$ and $(f, G)$ becomes $\left(f, G_{0}\right)^{2} /\left(G_{0}, I G_{0}\right)$ which is the same as would be calculated from (7).

We proceed now to prove the theorem. The first variation of (7) with $g=G$ when $c_{n}$ becomes $c_{n}+\delta c_{n}$ is

(10)

$$
\frac{\left\{2(f, G)\left(f, \sum_{n} G_{n} \delta c_{n}\right)(G, I G)-2(f, G)^{2}\left(I G, \sum_{n} G_{n} \delta c_{n}\right)\right\}}{(G, I G)^{2}} .
$$

Suppose now that equations (9) are satisfied. Then (10) vanishes if $(f, G)=(G, L G)$. Now $(f, g)=\left(f, \sum_{n} c_{n} G\right)=\left(L G, \sum_{n} c_{n} G\right)$ on account of (9); hence

$$
(f, G)=(G, L G)
$$

so that the first variation is zero. The equations (9) are thus sufficient for (7) to be stationary. 
Suppose now that the first variation is zero for independent $\delta c_{n}$.

Then

$$
(G, I G)\left(f, G_{n}\right)=(f, G)\left(L G, G_{n}\right) \quad(n=0,1, \ldots)
$$

These equations are homogeneous in the constants $c_{0}, c_{1} \ldots$. Hence if there is one solution there are any number of the form $\mathrm{Cc} c_{0}, \mathrm{Cc} c_{1} \ldots$ where $\mathrm{C}$ is a constant. There is therefore a solution in which

$$
(G, \ddot{I G})=(f, G) \text {. }
$$

Such a solution satisfies equations (9) and also the reciprocity theorem. The necessity for the equations has therefore been shown.

On account of (11) and (12), $A=(f, G)$ and the theorem has been proved. The corresponding theorem for the more general expression (6) will now be stated. No proof will be given since it runs parallel to that of l'heorem 1. We take $G^{\prime}$ to be the approximation to $\mathrm{g}^{\prime}$ and try

$$
G^{\prime}=\sum_{m} c_{m} G_{m}^{\prime}
$$

this series containing as many terms as (8). Then

Theorem 2 The necessary and sufficient conditions for the expressIon (6) with $g=G$ and $g^{\prime}=G^{\prime}$ to be stationary for small independent variations of $G$ and $G^{\prime}$ are

$$
\left(f, G_{m}^{\prime}\right)=\left(I G, G_{m}^{\prime}\right) \quad(m=0,1, \ldots)
$$

and

$$
\left(f^{\prime}, G_{m}\right)=\left(I G^{\prime}, G_{m}\right)
$$

$G$ and $G^{\prime}$ satisfy the reciprocity relation $\left(f^{\prime}, G\right)=\left(G, I G^{\prime}\right)=\left(f, G^{\prime}\right)$. The value of $A$ is either $\left(f, G^{\prime}\right)$ or $\left(f^{\prime}, G\right)$. 


\section{The accuracy of the approximation}

To determine a good approximation it is necessary to specify precisely what is meant by good. This involves stipulating the behavior of the operators and functions in some manner. We shall choose the behavior which is appropriate to a perturbation. Attention will be concentrated on the case $f=f^{\prime}$ so that $A=(f, g)$ and the reciprocity theorem takes the form $(f, g)=(L g, g)$.

Let $a$ be a parameter such that

$$
\text { for some range of } \begin{aligned}
a \\
I=I_{0}+a I_{1}+a^{2} I_{2}+\ldots \\
f=f_{0}+a f_{1}+a^{2} f_{2}+\ldots \\
g=g_{0}+a g_{1}+a^{2} g_{2}+\ldots \\
G=G^{0}+a G^{I}+a^{2} G^{2}+\ldots,
\end{aligned}
$$

(11) the only solution of $L_{0} h=0$ is $h=0$,

(ii1) $\left(h_{1}, L_{p} h_{2}\right)=\left(L_{p} h_{1}, h_{2}\right)$ for all $p$.

When $\alpha$ is small we are writing the operator $I$ as one which is slightly perturbated from $\mathrm{L}_{0}$. If a measures the ratio of the linear dinensions of the obstacle and the wavelength, and $L_{0}$ is the static operator, then we are considering the low-frequency behavior. It has been shown by Magms [5] that assumption (1) is justified for the problem of a low-frequency plane wave falling on a circular aperture.

If we substitute the formulas of (i) in (1) and equate the coefficients of powers of $\alpha$ the equations to determine $g$ are

$$
\sum_{r=0}^{p} L_{p-r} g_{r}=f_{p} \quad(p=0,1, \ldots) .
$$


If the inverse of the operator $\mathrm{L}_{0}$ is known, $\mathrm{g}$ can be found, in principle, correct to any power of $a$. Assumption (ii) ensures a unique solution.

The approximation $G$ is to be determined so that the reclprocity theorem

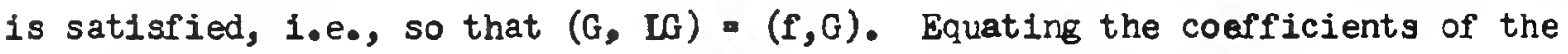
like powers of $a$ in the expansions of both sides, we obtain

$$
\sum_{r=0}^{p} \sum_{g=0}^{p-r}\left(G^{r}, L_{p-r-s} G^{k}\right)=\sum_{r=0}^{p_{0}}\left(G^{r}, f_{p-r}\right) \quad(p=0,1, \ldots)
$$

or

$$
\sum_{r=0}^{p} \sum_{s=0}^{p-r}\left(G^{r}, L_{p-r-s} a^{s}-L_{p-r-s} g_{s}\right)=0
$$

on account of (13).

Now we are going to approximate $(f, g)$ by $(f, G)$. Both quantities can be expanded in powers of $\alpha$ and the coefficients of $\alpha^{p}$ are the same if

$$
\sum_{s=0}^{p}\left(f p-s, G^{s}-g_{s}\right)=0
$$

Consequently the first $P$ terms of $(f, g)$ are given correctly by the approximation if (15) holds for all $\mathrm{p} \leq \mathrm{P}-1$.

Let us now suppose that $G^{\mathbb{M}}=g_{m}(m \leq M)$. Then, in (14), we can replace $G^{r}$ by $g_{r}$ in all the inner products in which $p-r \leq M$ for in these the second member is identically zero. Also $G^{r}$ may be replaced by $g_{r}$ in all inner products in which $r \leq M_{0}$. By this means $G^{r}$ is replaced by $g_{r}$ in all inner products in (14) provided that $p \leq 2 M+1$. Hence, after a use of assumption (iii), 


$$
\sum_{r=0}^{p} \sum_{s=0}^{p-r}\left(L_{p-r-s} g_{r}, G^{s}-g_{g}\right)=0 \quad(p \leq 2 M+1)
$$

or

$$
\sum_{s=0}^{p} \sum_{r=0}^{p-s}\left(I_{p-r-s} g_{r}, a^{s}-g_{s}\right)=0 \quad(p \leq 2 M+1)
$$

on interchanging the order of summation. This equation becomes, on taking account of (13),

$$
\sum_{s=0}^{p}\left(f p-s, G^{s}-g_{g}\right)=0 \quad(p \leq 2 M+1)
$$

which is the same as (15). Thus if $G^{m}=g_{m}(m \leq M)$ the first $2 M+2$ terms of $(f, g)$ are given correctly by the approximation.

If we carry out a similar analysis with $p=2 M+2$ we find

$$
\sum_{s=0}^{2 M+2}\left(f_{2 M+2-s^{2}}, G^{S}-g_{g}\right)+\left(G^{M+1}-g_{M+1}, L_{0} G^{M+1}-L_{0} g_{M+1}\right)=0
$$

so that the $(2 M+3)$ rd term of $(f, g)$ is given correctly only if

$$
\left(G^{M+1}-g_{M+1}, L_{0} G^{M+1}-I_{o} g_{M+1}\right)=0
$$

This result enables us to obtain some information which is particularly applicable to the low-frequency behavior. At low frequencies, where $L_{\circ}$ is the static operator and $\alpha$ is purely imaginary, the following additional assumptions hold in most scattering problems

(iv) $I_{p}$ operating on a real function produces a real function for all $p$;

(v) If $h$ is real and $\left(h, L_{0} h\right)=0$ then $h=0$,

(v1) $f_{p}$ is purely real for all $p$. 
With assumptions (iv) and ( $\nabla 1$ ) it is easy to see that all $\mathrm{g}_{\mathrm{p}}$ and $G^{p}$ are real. Hence if $(16)$ holds, assumption $(v)$ shows that $G^{M+1}=g_{M+1}$. In particular, this applies if $M=-1$.

Consequently we have shown:

Theorem 3 The sufficient conditions for $(f, G)$, $G$ satisfying the reciprocity relation, to give correctly the first $2 \mathrm{M}+2$ terms of the power series expansion of $(f, g)$ are

$$
G^{m}=g_{m} \quad(m \leq M)
$$

when assumptions (i) to (iii) are satisfied. If, in addition, assumptions (iv) to ( $v i$ ) hold, the conditions are also necessary.

The corresponding theorem for the more general case is not so powerful. It is

Theorem 4 The sufficient conditions for $\left(f^{\prime}, G\right)$ or $\left(f, G^{\prime}\right), G$ and $G^{\prime}$ satisfying the reciprocity relations, to give correctly the first $2 \mathrm{M}+2$ terms of the power series expansion of $\mathrm{A}$ are

$$
G^{m}=g_{m}, G^{\prime m}=g_{m}^{\prime} \quad(m \leq M)
$$

when assumptions (1) to (iii) hold with appropriate addition to (i) for $G^{\prime}$ and $g^{\prime}$.

The proof is similar to that for Theorem 3 and will not be given.

4. The low-freouency behavior

In this section we shall consider the case $f=f^{\prime}$ when assumptions (i) to ( $\nabla i$ ) hold. These assumptions are spectally applicable to the low-frequency behavior and we shall make particular reference to the process employed by Levine and Schwinger ${ }^{[2]}$. Assume that $G=\sum_{m=0}^{n} c_{m} G_{m}$ where the $G_{m}$ are real, independent of a and defined in a similar way to those of Theorem 1 . The constants $c_{m}$ are 
determined by equations (9). One hopes to obtain an approximation which is reasonable over the whole frequency band by a suitable choice of $G_{m} \cdot$ By means of Theorem 3 we can say what this choice must be for the low-frequency end. While we cannot guarantee from this work that the choice will also be appropriate for the high frequency end, we can say that, at any rate, it is not precluded.

Suppose that we desire that this approximation $G$ gives the first $2 n+2 N+2$ terms of $(f, g)$ correctly at low frequencies, $N$ being an integer. If, in particular, $\mathrm{n}=0$ we want to make $\mathrm{N}$ as Large as possible. Now, when the first $2 n+2 N+2$ terms of $(f, g)$ are correct, it follows from Theorem 3 that $G^{m}=g_{m}(m \leq n+N)$. But $G^{m}$ can be expressed in terms of $G_{0}, G_{1}, \ldots, G_{n}$. Hence the first $2 n+2 N+2$ terins of $(f, g)$ can be correct only if $G_{0}, G_{1}, \ldots, G_{n}$ are linear combinations of $g_{0}, g_{1}, \ldots, g_{n+N}$ or, alternatively, if $g_{0}, g_{1}, \ldots, g_{n+N}$ are linear combinations of $G_{0}, G_{1}, \ldots, G_{n}$.

If, in addition, we require the first $2 n+2 N+2$ terms to be correct for all $n$ then, on taking $n=0, g_{0}, g_{1}, \ldots, g_{N}$ must be constant multiples of $G_{0}$. Taking $n=1$, we deduce that $\mathrm{g}_{\mathrm{N}+1}$ must be a linear combination of $G_{0}$ and $G_{I} \cdot$ From $n=2, g_{N+2}$ must be a linear combination of $G_{0}, G_{1}$ and $G_{2}$ and the process continues.

Thus the maximum number of terms which can be obtained by a one-element approximation to $G(n=0)$ is determined by the first $g_{n}$ which is not a constant multiple of $\mathrm{g}_{0}$.

Consider now the application of these results to the problem considered by Levine and Schwinger. In that problem $f_{1}=0, I_{1}=0$, so that $g_{1}=0$, but $g_{2}$ is not a constant multiple of $g_{0^{*}}$ Hence $N=1$ and the maximum number of terms of $(f, g)$ to be obtained from the approximation $G=c_{0} G_{0}$ is 4 and can be obtained only by choosing $G_{0}=g_{0^{\circ}}$. From $G=c_{0} C_{0}+c_{1} G_{1}$ we can obtain 6 terms provided 
that $G_{1}$ is a linear combination of $g_{0}$ and $g_{2}$ Simllar 15 , a suitable choice of $G_{2}$ will give 8 terms of $(f, g)$ and so on. The choice of $G_{n}$ made by Levine and Schwinger is in fact the one dictated by the above accuracy considerations. Of course it is not necessary to make their particular choice of $G_{1}$ to ensure low-frequency accuracy; we can choose any linear combinations of $\mathrm{g}_{0}$ and $\mathrm{g}_{2}$ so long as both are included. Similar remarks apply to any $G_{n}$ and, in general, we would choose those that are most convenient for dealing with equations (9). Finally, there is one thing to be careful of in the use of Theorem 3. The statement of accuracy in this theorem depends upon $\mathrm{L}$ and therefore upon the accuracy with which $I$ is approximated. Thus, when the inner products in (9) have to be evaluated approximately (as often happens), care must be taken that this evaluation does not vitiate Theorem 3. Onfy if the constants in (9) are obtained correct to $O\left(a^{2 M+1}\right)$ at the low-frequency end is Theorem 3 available.

\section{Bounds for the error}

Although the analysis of the preceding section indicates how the trial function must be chosen, it does not give a precise estimate of the error nor does it state the range of a for which the approximation is good. In practice, it may well be more desirable to use an approximate field for which it is possible to say that $(f, g)$ is estimated correctly to (say) $10 \%$ for a certain range of wavelength than to insist that the field satisfies the reciprocity theorem and gives (say) $1 \%$ accuracy over an unknown range. We shall now show how an estimate of the error can be made in certain circumstances.

Let $Y=g-G$ where $G$ is some approximatior to $g$. Then

$$
(f, \gamma)=(I g, \gamma)=(g, L \gamma)
$$

or

$$
(f, g)=(f, G)+(G, L Y)+(Y, L Y) .
$$


Since

$$
L Y=f-L G
$$

the second term may also be written as $(G, f-I G)$. It is easy to see from (17) that $2(f, g)-(g, I g)$ is a stationary expression for $(f, g)$.

If $G$ satisfies the reciprocity theorem the second term in (17) is zero and the error made by the approximation is $(Y, I Y)$. This result sometimes enables us to state the sign of the error. For example, if all quantities are real and $L$ is positive definite, we see that $(f, G)$ is a lower bound to $(f, g)$.

Even if $G$ does not satisfy the reciprocity theoren the only term which cannot be calculated in (17) is the third because the second can always be evaluated by means of (18). An estimate of the goodness of the approximation therefore requires only an estimate of the third term in (17); this estimate depends essentially on being able to deduce properties of $\gamma$ from a knowledge of LY. There does not appear to be any method which we can say will be generally applicable because the operator and functions occurring are usually complex but we shall give two methods which are available in suitable circumstances.

Firstiy, suppose that it is possible to say

$$
\left|\left(h_{1}, h_{2}\right)\right|^{2} \leq\left(h_{1}, h_{1}^{*}\right)\left(h_{2}, h_{2}^{*}\right)
$$

where the star indicates a complex conjugate and suppose, further, that there is a $\mu>0$ such that

$$
\left(L Y, L^{*} \gamma^{*}\right) \geq \mu\left(\gamma, \gamma^{*}\right)
$$

for all $\gamma$ which can arise. Then

$$
|(Y, L Y)| \leq \frac{I}{\sqrt{\mu}}\left(\operatorname{Ir}, L^{*} \gamma^{*}\right) .
$$


Since $\mu$ and LY ar both known this inequality gives an estimate of the error. We may also determine the accuracy of the other approximations. For $\left(f^{\prime}, g\right)=\left(f^{\prime}, G\right)+\left(f^{\prime}, Y\right)$ and

$$
\left|\left(f^{8}, \gamma\right)\right|^{2} \leq \frac{I}{\mu}\left(f^{\prime}, f^{\prime *}\right)\left(L Y, L^{*} \gamma^{*}\right)
$$

all quantities are known on the right hand side of (19).

A second method, related to that used by Kato[6] in problems of atomic coliisions, deperds upon adjusting the incident field until a prescribed scattered amplitude is obtained. The scattered amplitude corresponding to the originel incident field can then be deduced immediately. Let $\nu$ be a complex constar" such that, if

$(20)$

$$
V f=I g^{\circ} \text {, }
$$

$$
\left(f, g^{0}\right)=1
$$

Since $g^{\circ}=\nu g$ we see that $(f, g)=\frac{l}{\nu}$. Consider now $G$ chosen so that

$$
(f, G)=1
$$

Let $G^{0}=\nu^{\prime} f-(I-1) G=\nu^{\prime} f$ - HG where $\nu^{\prime}$ is a complex constant. $G^{0}$ is considered as an approximation to $\mathrm{g}^{\circ}$. Then

$$
\begin{aligned}
\left(g^{\circ}, G-G^{\circ}\right) & =\left(g^{\circ}, L G-\nu^{\prime} f\right)=\left(L_{g}^{\circ}, G\right)-\nu^{\prime} \\
& =\nu-\nu^{\prime}
\end{aligned}
$$

on account of $(20,(21)$ and $(22)$. Hence

$$
\nu=\nu^{\prime}+\left(G^{\circ}, G-G^{0}\right)+\left(g^{0}-G^{0}, G-G^{0}\right) .
$$


-15 -

We can regard the first two terms as giving an approximation to $\nu$, the third being the error.

To estimate this error, we introduce the equation

(24)

$$
(1+\lambda) M \phi+\phi=\bigwedge f
$$

where $(I+\lambda)(f, \varnothing)=0$ and $\Lambda$ is a nonzero constant to be chosen lat as. assume that nonzero $\varnothing$ can be obtained only for a discrete set of real. which $\lambda_{n}$ is a typical one, $\varnothing_{n}$ being the corresponding solution. Since

$$
\left(\phi_{\mathrm{n}}, \phi_{\mathrm{m}}\right)=-\left(\lambda_{\mathrm{m}}+I\right)\left(\phi_{\mathrm{n}}, M \phi_{\mathrm{m}}\right)=-\left(\lambda_{\mathrm{m}}+1\right)\left(M \phi_{\mathrm{n}}, \phi_{\mathrm{m}}\right)=\frac{1+\lambda_{\mathrm{m}}}{1+\lambda_{\mathrm{n}}}\left(\phi_{\mathrm{n}}, \phi_{\mathrm{In}}\right.
$$

we choose $\bigwedge$ for each $\lambda_{n}$ so that

$$
\left(\not \not_{m}, \varnothing_{n}\right)=\delta_{n m}
$$

where $\delta_{n m}=0(n \neq m),=I(n=m)$. It will be assumed that the $\emptyset_{n}$ form a complete set.

Define the constants $a_{n}$ and $b_{n}$ by

$$
\left(G-G^{0}, \varnothing_{n}\right)=a_{n}
$$

and

$$
\left(g^{\circ}-G^{0}, \emptyset_{n}\right)=b_{n} .
$$

Then

$$
\begin{aligned}
b_{n} & =\left(\nu f-M g^{0}-\nu^{\prime} f+M G, \phi_{n}\right) \\
& =\left(M\left(G-g^{0}\right), \phi_{n}\right)
\end{aligned}
$$

because of (24); thus 


$$
\begin{aligned}
b_{n} & =\left(G-g^{0}, M \phi_{n}\right) \\
& =-\frac{1}{1+\lambda_{n}}\left(G-g^{0}, \phi_{n}\right)
\end{aligned}
$$

on account of (21) and (22). Also

$$
\begin{aligned}
a_{n} & =\left(I G-I Q^{\circ}, \emptyset_{n}\right) \\
& =\left(G-g^{\circ}, L \emptyset_{n}\right) \\
& =\frac{\lambda_{n}}{1+\lambda_{n}}\left(G-g^{\circ}, \phi_{n}\right) \\
& =-\lambda_{n} b_{n} .
\end{aligned}
$$

Therefore

and

$$
\left(g^{0}-G^{0}, G-G^{0}\right)=\sum a_{n}^{a} b_{n}=-\sum \frac{1}{\lambda_{n}} a_{n}^{2}
$$

$$
\left(G-G^{0}, G-G^{0}\right)=\sum a_{n}^{2} \text {. }
$$

If, now, we can say that $a_{n}$ is real for all $n$, it follows that

$$
-\frac{I}{\lambda_{+}}\left(G-G^{0}, G-G^{0}\right) \leq\left(B^{0}-G^{0}, G-G^{0}\right) \leq-\frac{1}{\lambda_{-}}\left(G-G^{0}, G-G^{0}\right)
$$

where $\lambda_{+}$is the smallest positive $\lambda_{n}$ and $\lambda_{-}$is the negative $\lambda_{n}$ of smallest absolute value. (Note that $\lambda_{n}$ cannot equal zero for any $n_{0}$ ) Since $G^{0}$ is known in terms of $\nu^{\prime}$ and $G$ we have obtained an estimate for the error in using the first two terms of (23) as an approximation to $\nu$. The accuracy of $G^{0}$ as an approximation to $\mathrm{g}^{0}$ may also be obtained from 
$-17-$

$$
\begin{aligned}
\left(g^{0}-G^{0}, g^{0}-G^{0}\right) & =\sum b_{n}^{2} \\
& \leq \max \left(\frac{1}{\lambda_{+}},-\frac{1}{\lambda_{-}}\right)\left(G-G^{0}, G-G^{0}\right) .
\end{aligned}
$$

This enables us to determine the error in quantities such as $\left(f^{\prime}, g^{\circ}\right)$.

In practice this method may not be at all easy to apply because of the many restrictions. We have first to verify, in any particular problem, that the $\varnothing^{\prime} s$ satisfy the conditions imposed and then that $G$ and $\nu^{\prime}$ can be chosen so that $a_{n}$ is real. (The latter condition is, at any rate, complied with when $f$ and $L$ are real.) Then $\lambda_{+}$and $\lambda_{-}$(or bounds for them) must be determined. Fortunately, great accuracy is not required here because we make $\left(G-G^{\circ}, G-G^{\circ}\right)$ as small as possible by taking the best choice of $\nu^{\prime}$ among those values which keep $a_{n}$ real. 
References

[1] Volterra, v. - Sopra un Problema die Elettrostatica; II Nuovo Cimento, 16, 49-57 (1884).

[2] Levine, H. and Schwinger, J. - On the theory of diffraction by an aperture in an infintte plane screen. I. 3 Phys. Rev., 74, 958-74 (1948).

[3] Kohn, W.

- Variational methods in muclear collision problems; Phys. Rev., 74, 1763-72 (1948).

[4] Lippmann, B.A. - Variational formulation of a grating problem, Rept. N.D.A. 18-8 Nuclear Derelopment Associates, Inc., White Plains, N.Y. (1952).

[5] Magnus, W.

- Infinite matrices associated with diffraction by an aperture; Quart. App. Math., 11, 77-86 (1953). New York University, Institute of Mathematical Sclences, Division of Electromagnetic Research, Research Report No. EM-32, May, 1951.

[6] Kato, T.

- Upper and lower bounds of scattering phases; Progress of Theor. Phys., 6, 394-407 (1951). 



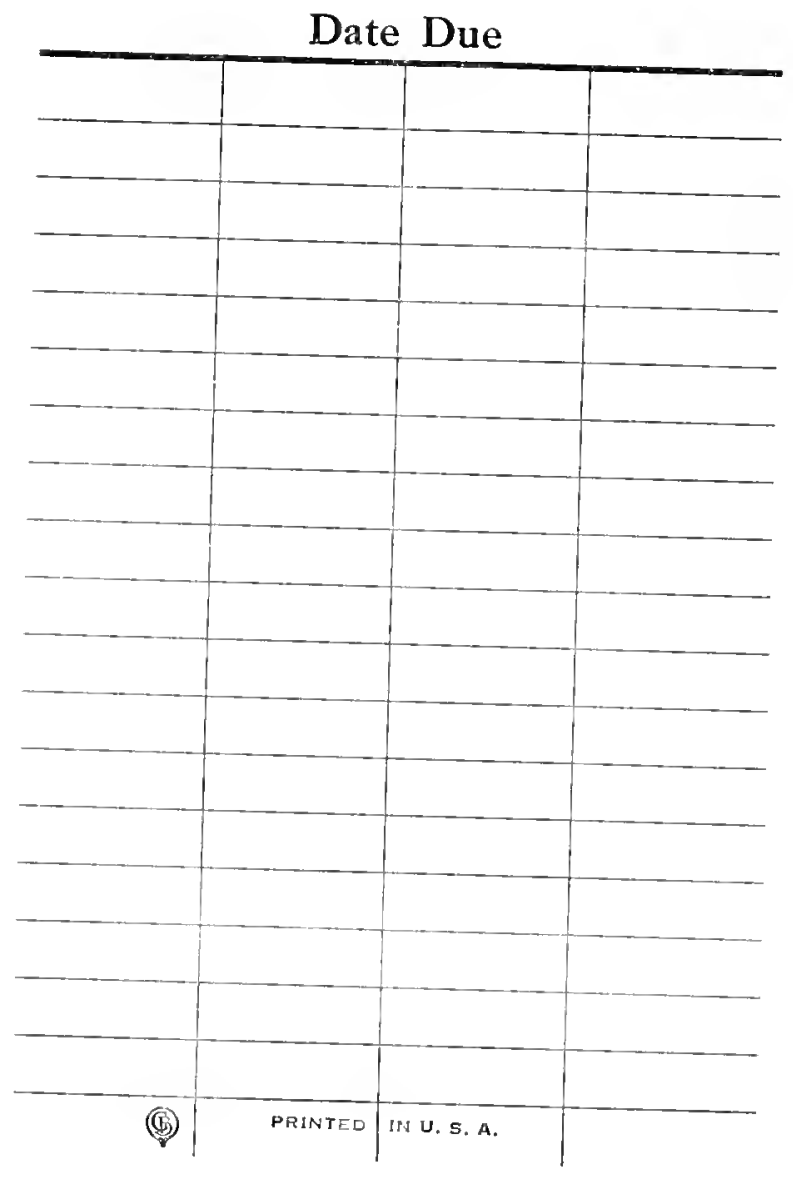


NYTI

iin

Jores

A critique of the variational method in scetter ine rroblems.

NYU

Ein

78

Jones

is eriticue of the varoiationad

notiod ir seattening rnoolem 



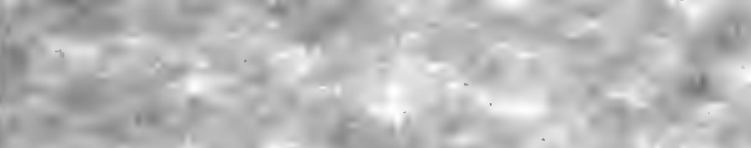

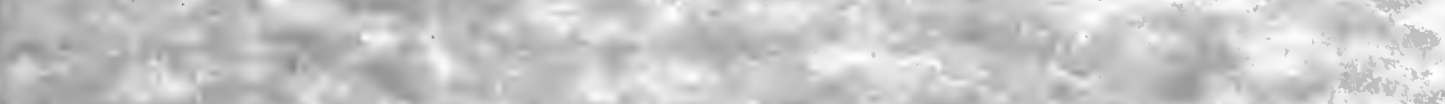

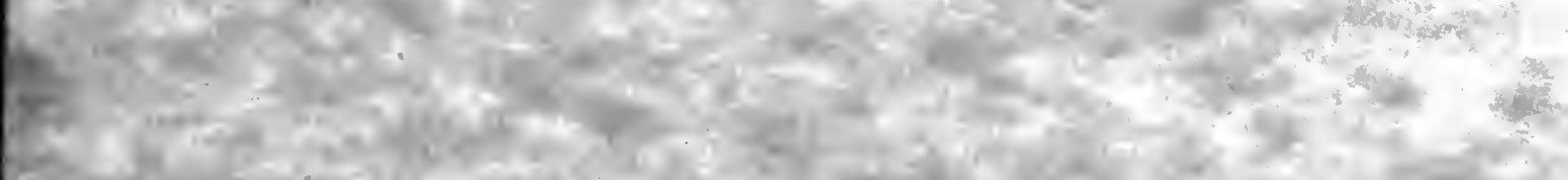

1.

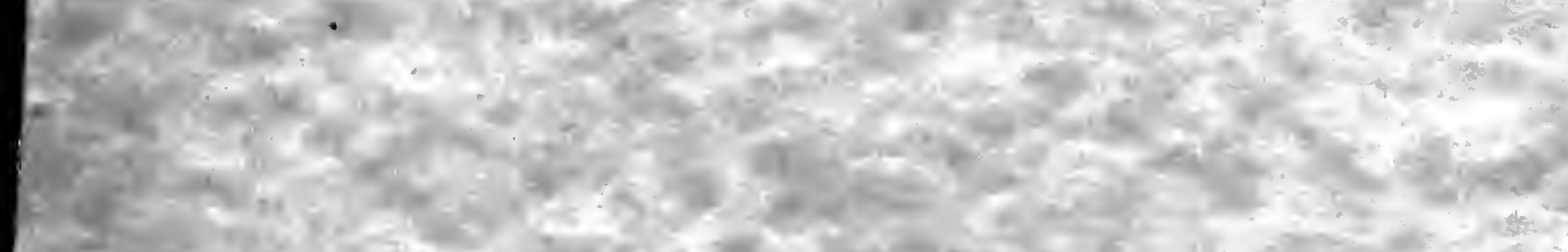

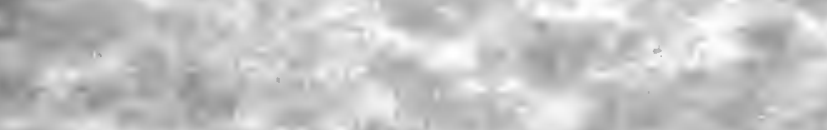

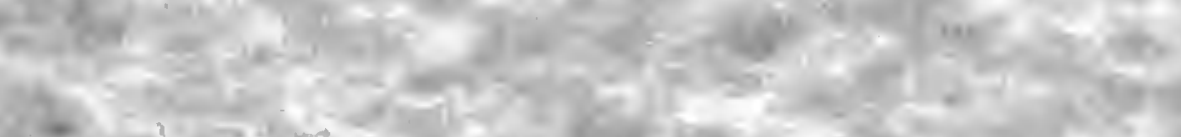

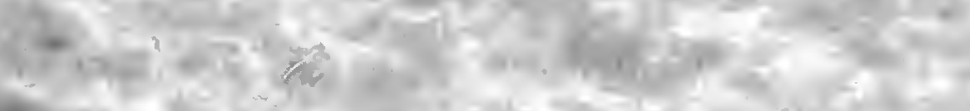

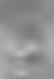

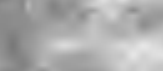

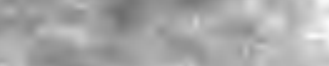

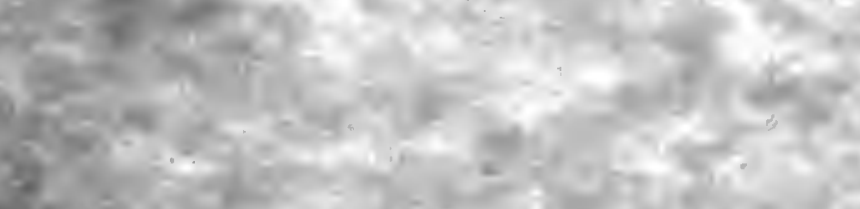

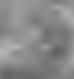

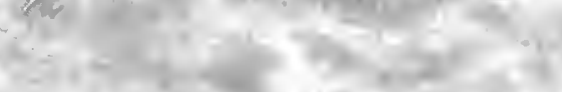

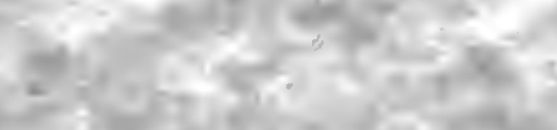

$(-)^{2}$

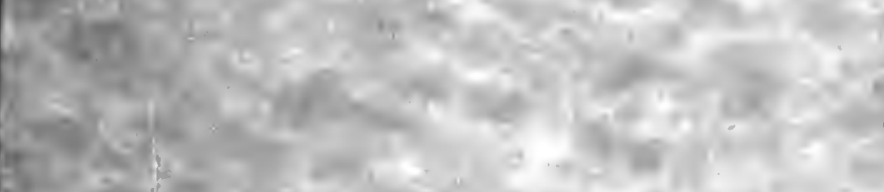

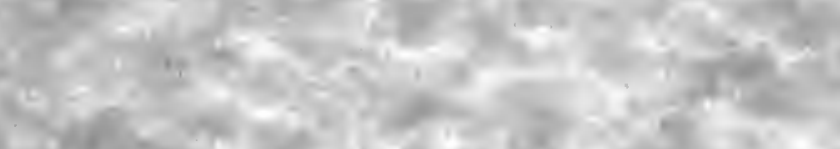

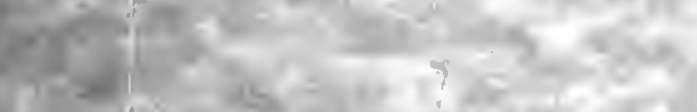

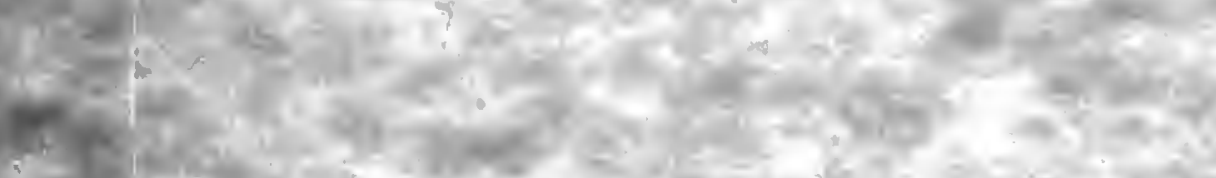

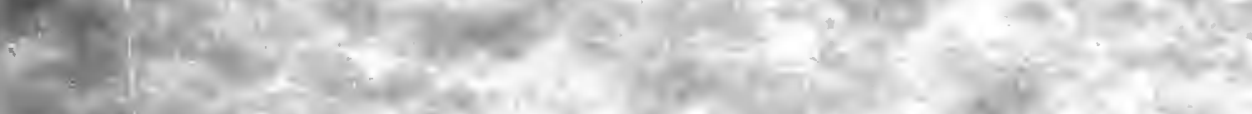

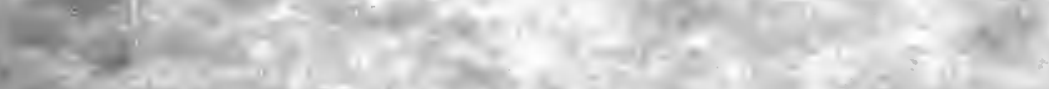

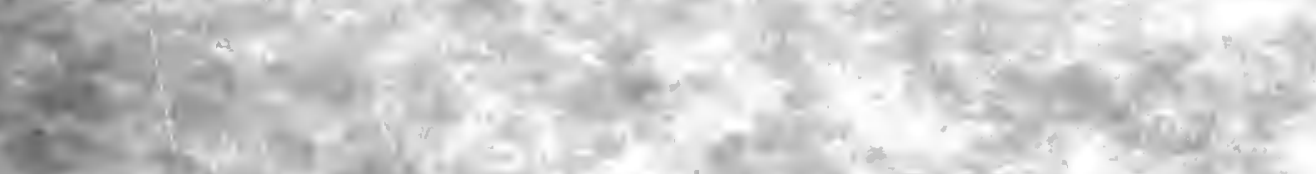

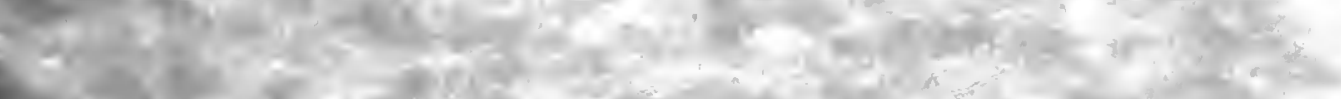

6.

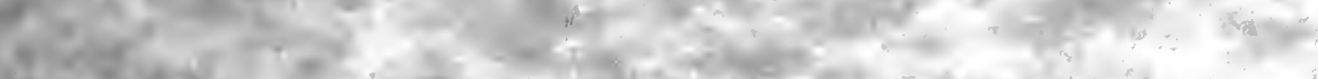

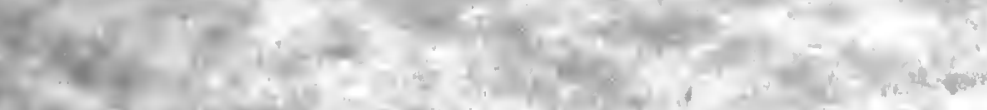

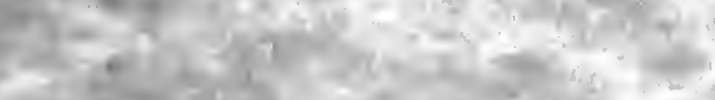

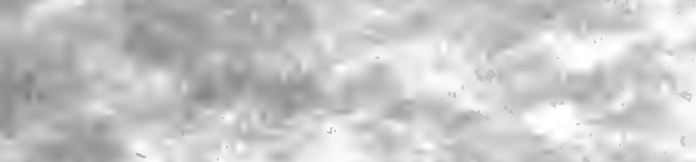

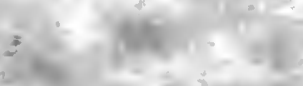

Nents 


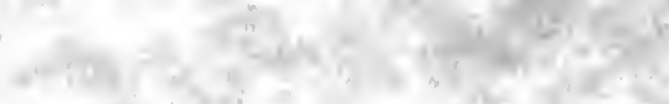

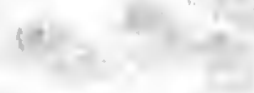

H.

$\therefore$.

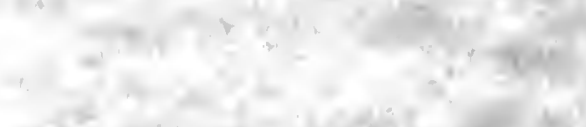

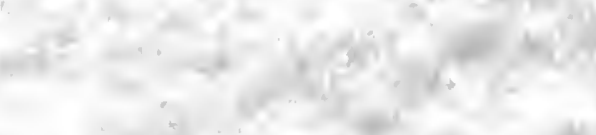

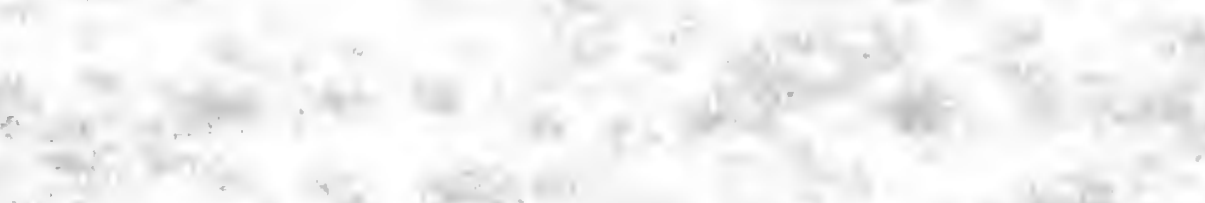

